\title{
Sensing AND Reference PAds For INTEGRATED OPTICAL IMMUNOSENSORS
}

\author{
Jürg Dübendorfer ${ }^{\dagger}{ }^{\text {Rino E. Kunz }}{ }^{\dagger}$ E. Schürmann, ${ }^{*}$ Gert L. Duveneck, ${ }^{\ddagger}$ \\ and Markus Ehrat ${ }^{\ddagger}$ \\ ${ }^{\dagger}$ Paul Scherrer Institute, Badenerstrasse 569, CH-8048 Zurich, Switzerland; "Novartis Pharma AG, \\ Bioanalytical Research, P.O. Box, CH-4002 Basle, Switzerland \\ (Paper JBO-148 received Mar. 20, 1997; revised manuscript received June 16, 1997; accepted for publication July 2, 1997. )
}

\begin{abstract}
For integrated optical immunosensors, a much more reliable interpretation of the sensor output signal can be achieved by introducing reference pads near the sensing pads, in order to separate specific from nonspecific effects. The results of theoretical and experimental investigations are reported for immunosensors based on measuring changes of the effective refractive index due to the binding of analyte molecules. The emphasis is on the correction for variations of the light wavelength, the angle of incidence, and the temperature for integrated optical grating coupler chips. Using the binding of rabbit immunoglobulin to immobilized protein A as a regenerable model bioaffinity system, the influence of consecutive assay cycles on the two pads has been investigated. Experiments have been performed using sensor chips consisting of $\mathrm{TiO}_{2}$ waveguiding films on fused silica and on replicated polycarbonate substrates. (ㄷ 1997 Society of Photo-Optical Instrumentation Engineers. [S1083-3668(97)00404-8]
\end{abstract}

Keywords integrated optical sensors; evanescent wave sensors; replicated integrated optical immunosensors, on-chip referencing.

\section{INTRODUCTION}

Many evanescent wave sensors are based on monitoring effective index changes caused by the adsorption of analyte molecules on the transducer. The fabrication of sensing pads suitable for creating a great variety of such miniature integrated optical (IO) immunosensors with high sensitivities was reported earlier. ${ }^{1}$ The sensing schemes are mainly based on interferometry ${ }^{2-4}$ and on grating couplers. ${ }^{1,5,6}$ However, the transfer of the highly sensitive laboratory systems to sensors for practical use is not straightforward, since the sensor output signal is also strongly affected by nonspecific effects and external disturbances, originating, for example, from aging of chemical layers, changes in temperature, light wavelength, angle of incidence, and reagent refractive index.

In this paper, an approach is presented for achieving more reliable interpretations of the sensor output signals by introducing reference pads located near the sensing pads of high-sensitivity grating coupler sensor chips. Results are reported for fluctuations of the light wavelength, the angle of incidence, and the temperature as important examples of nonspecific signal disturbances that are relevant for many practical applications. Figure 1 shows two sensor types and the respective methods

Address all correspondence to Jürg Dübendorfer. Tel: 411492 6350; Fax: 411491 0007; E-mail: juerg.duebendorfer@psi.ch

Current affiliation of Rino E. Kunz: CSEM Centre Suisse d'Electronique et de Microtechnique SA, Badenerstrasse 569, CH-8048 Zurich, Switzerland. for accomplishing true on-chip referencing: (a) a grating coupler sensor chip as an input or output coupler $^{7-9}$ and (b) a Mach-Zehnder interferometer. $^{2,10}$ Experimental results are presented for the referenced input grating coupler sensing scheme [see Figure 1(a)] where a reference pad $R$ is located close to the sensing pad $S$. In Sec. 2, the principles of referencing in a simple biochemical model system, namely, the binding of rabbit immunoglobulin (r-IgG) to immobilized protein $\mathrm{A}$, are presented. Some fundamental theoretical and experimental aspects are described in Sec. 3. In Sec. 4, experimental results obtained for high-sensitivity sensors consisting of $\mathrm{TiO}_{2}$ films on fused silica $\left(\mathrm{SiO}_{2}\right)$ and on replicated polycarbonate (PC) substrates are presented and discussed.

\section{REFERENCING IN AN IMMUNOASSAY}

Biochemical affinity assays are well suited for biochemical and clinical analysis ${ }^{11}$ due to their excellent specificity. Biochemical recognition elements, such as antibodies, receptors, or enzymes are used for selective detection of a target analyte among hundreds or thousands of other substances. As a simple biochemical model system for the sensing pad development, the binding of rabbit immunoglobulin (r-IgG) to immobilized protein A was chosen. The system can be used as the basis for sandwich immunoassays, where $\operatorname{IgG}$ bound to protein A acts as the primary antibody for the analyte.

1083-3668/97/\$10.00 @ 1997 SPIE 


\section{$: S$}

(a)

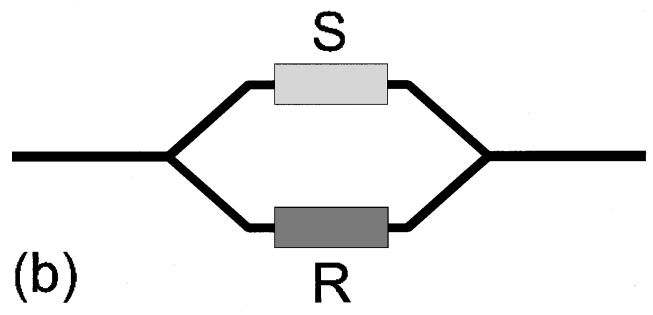

Fig. 1 Examples of 10 sensor types and possible arrangements of sensing pads $S$ and reference pads $R$. (a) Grating coupler sensor chip. (b) Mach-Zehnder interferometer.

An advantage of using evanescent wave sensors to detect effective refractive index changes of a guided mode is that only binding events near the transducer surface are detected. The labeling of analytes with fluorescent or radioactive markers is not necessary. High sensitivities of these sensors can be obtained when the field amplitudes of the guided modes are high in the sensing layer. ${ }^{12}$

Figure 2(a) shows schematically the transducer response of an ideal immunoassay. The sensing layer consists of two parts: protein A, immobilized on the waveguide surface and acting as the recognition element, and the IgG adsorbate element. A typical assay cycle starts with an equilibration of the sensor in buffer solution. Then, r-IgG analyte solution is supplied for 10 to $30 \mathrm{~min}$ to the sensor, depending on the analyte concentration. Before performing the regeneration step, buffer solution is supplied again for studying the dissociation of the formed bioaffinity complexes. The regeneration (not shown in Figure 2) removes the IgG without removing the protein $\mathrm{A}$, and without reducing the functionality of the protein.

The transducer response of an assay disturbed by external or nonspecific effects $E 1$ and $E 2$ is shown schematically in Figure 2(b). The corresponding response of the reference pad is shown in Figure 2(c). The surface of the reference pad is passivated by means of a reference layer to avoid binding of the analyte. Consequently, in the ideal case, the reference pad does not respond to the supply of the analyte solution, but only to the nonspecific effects E1 and E2. Hence, the disturbed transducer response of Figure 2(b) can be corrected by subtracting the reference transducer response from the disturbed sensing pad signal, yielding the ideal assay curve of Figure 2(a), even for nonideal conditions.
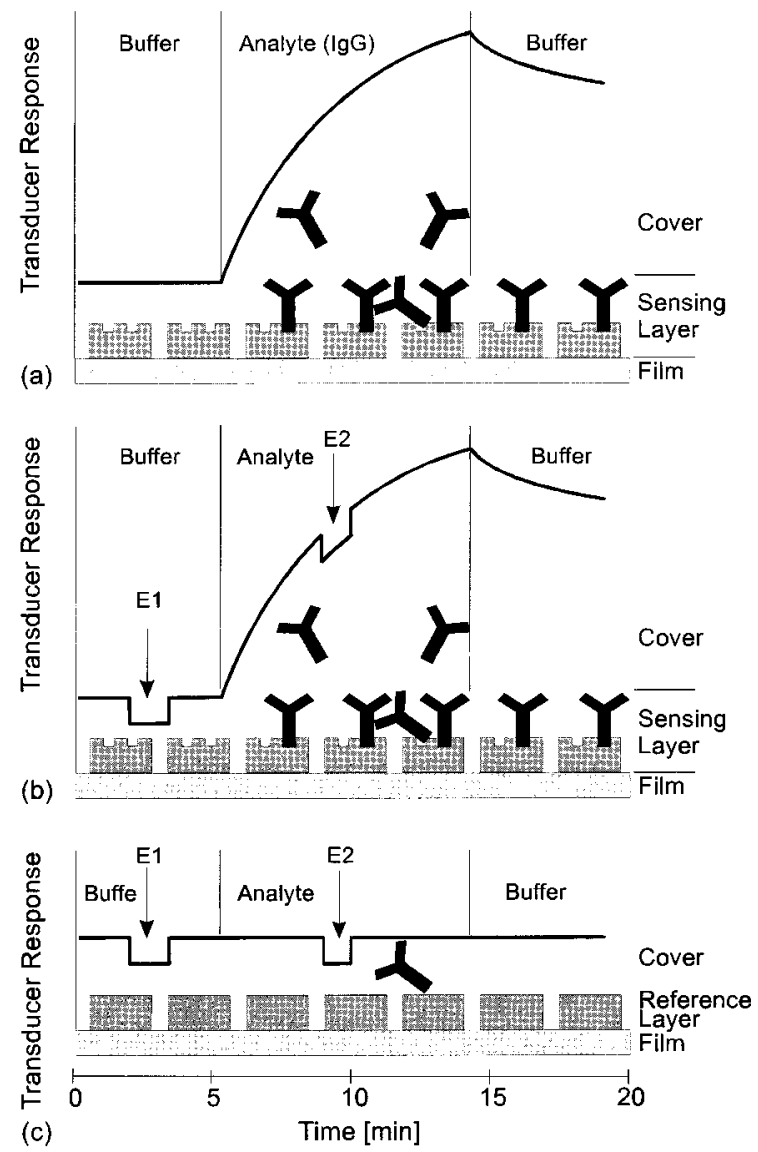

Fig. 2 Idealized transducer response for the binding of $r-\lg G$ to immobilized protein A. (a) Ideal assay cycle. (b) Response of an externally or nonspecifically disturbed assay. (c) Corresponding response of the reference pad having no recognition elements.

\section{IMPORTANT THEORETICAL AND EXPERIMENTAL ASPECTS}

Figure 3 shows the grating coupler sensor chip configuration used for performing the experiments. The configuration is based on a multilayer system consisting of a high refractive index waveguiding film with thickness $h_{f}$ and refractive index $n_{f}$ on a

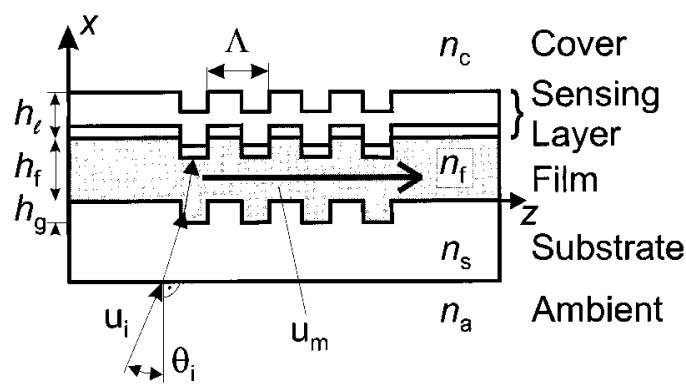

Fig. 3 Grating coupler sensor chip in an input coupler sensing scheme. $n_{a}, n_{s}, n_{f}, n_{c}$ : refractive indices of ambient, substrate, film, and cover medium, respectively; $h_{g}$ : grating depth; $h_{f}$ : film thickness; $h_{l}$ : sensing layer thickness; $\Lambda$ : grating periodicity; $u_{i}$ : incident wave; $\theta_{i}$ : angle of incidence; $u_{m}$ : guided wave. 
planar substrate with refractive index $n_{s}$. The grating structures were fabricated by means of photolithography and dry etching techniques into fused silica substrates and by hot embossing into polycarbonate sheets by means of an $\mathrm{Ni}$ shim for replicated plastic chips. The waveguides were fabricated by depositing a thin $\mathrm{TiO}_{2}$ film onto the previously structured substrates. The film deposition process reproduces the grating structure, yielding a multiply stacked grating. Details on fabrication and characterization can be found in Refs. 13 and 14. The recognition layer and the adsorbate layer form together the sensing layer with thickness $h_{l}$ and refractive index $n_{l}$. The cover medium is the aqueous buffer solution. The waveguiding film thickness $h_{f}$ is optimized for maximum sensitivity $\partial N / \partial h_{l},{ }^{1,12}$ for this sensor configuration. Here $N$ denotes the effective refractive index. Its dependence on the waveguide parameters and on the sensing layer thickness $h_{l}$ cannot be represented by an analytical equation. Besides using commercial computer programs, some methods and useful expressions for calculating $N$ can be found in Refs. 12 and 15-17.

For preparing the sensing layer, the sensor chips were first silanized in gas phase at room temperature. Then two adjacent regions on the grating, separated by about $2 \mathrm{~mm}$ [see Figure 1(a)], were prepared in different ways. Only the sensing region $\mathrm{S}$ was incubated with protein $\mathrm{A}(1 \mathrm{mg} / \mathrm{ml}$, molecular weight $\approx 41,000 \mathrm{Da}$ ) by applying a small drop for $2 \mathrm{hr}$. In a next step, the chip was rinsed with phosphate buffer solution (PBS, pH 7.2), containing $1 \mathrm{mg} / \mathrm{ml}$ bovine serum albumin (BSA, molecular weight $\approx 67,500 \mathrm{Da}$ ) in a flow directed from the reference region $R$ toward the sensing region $S$. This step resulted in the formation of a BSA reference layer and simultaneously in blocking of nonspecific binding sites on the sensing pad. After the chip was rinsed, it was mounted in the test cell, and BSAcontaining buffer solution was supplied for at least another $30 \mathrm{~min}$, for further blocking of the sensing pad and completing the forming of the BSA reference layer. "Pollution" between the sensing and reference pads was avoided by choosing the flow direction always going from the reference pad toward the sensing pad (Figure 4). All the solvents and chemicals used for the experiments were analytical grade or chemically pure. Protein A and r-IgG were obtained from Sigma (St. Louis, Missouri). All solutions, except for sensor regeneration by glycine buffer, were prepared from one stock buffer solution.

The performance of the sensor chips was tested in an input grating coupler sensing scheme (Figure 3). The grating was illuminated by a plane wave $u_{i}$ at an angle of incidence $\theta_{i}$ measured in the ambient medium with refractive index $n_{a}=1$ (air). A guided mode $u_{m}$ is excited with maximum coupling efficiency if the grating coupler resonance condition

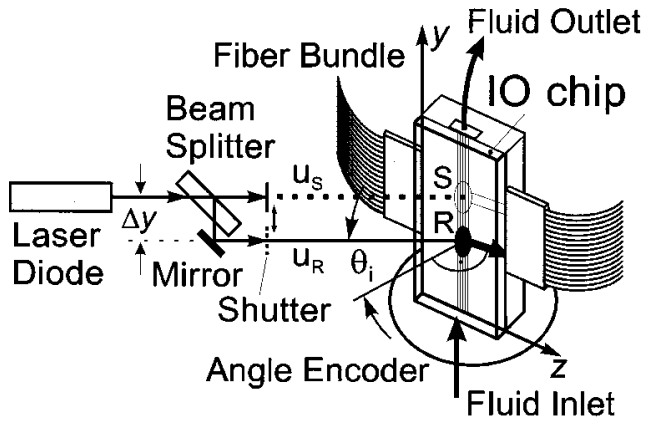

Fig. 4 Experimental setup for referenced input grating coupler measurements.

$$
N=n_{a} \sin \left(\theta_{i}\right)+m_{g} \lambda / \Lambda
$$

is fulfilled, where $m_{g}$ is the grating diffraction order, $\lambda$ denotes the wavelength of light in air, and $\Lambda$ is the periodicity of the grating.

The experimental setup is shown in Figure 4. The sensor chip was mounted in the flow-through cell with about a 20- $\mu$ l sample volume, and fluid inand outlets. The collimated beam of a laser diode (Sharp, LT026MD0, $\lambda=785.1 \mathrm{~nm}$ ) was split into two parallel beams $u_{S}$ and $u_{R}$ with an offset $\Delta y$ $\approx 2 \mathrm{~mm}$, using a beamsplitter and a mirror. These two input beams were multiplexed with a shutter for achieving a quasi-simultaneous readout at the two spots of the sensing pad $S$ and of the reference pad R [Figure 1(a)]. The resonance angle $\theta_{i}$ was obtained by angular scans using a high-resolution angle encoder (Heidenhain, RON 905, AWE 1024). During the scans, the excited mode power was measured by means of two fiber bundles connected to a single photodetector and subsequent curve fitting with a Lorentzian curve. The effective index $N$ was then calculated using Eq. (1). For practical reasons, the results will be reported in terms of changes $\Delta h_{l}$ in the sensing layer thickness $h_{l}$. This equivalent layer thickness $h_{l}$ is the numerical value of a single layer model with $n_{l}(\lambda=785 \mathrm{~nm})=1.45$, which yields the same effective index $N$ as observed in the experiment. ${ }^{1}$ The changes $\Delta h_{l}$ in the sensing layer thickness correspond to an average over a fractional monolayer of bound r-IgG. Changes $\Delta \Gamma$ of the surface mass coverage $\Gamma$ can be calculated using $h_{m}=8.5 \mathrm{~nm}$ for the thickness and $\Gamma_{m}=5 \mathrm{ng} / \mathrm{mm}^{2}$ for the surface mass coverage of an IgG monolayer ${ }^{3,18}$ adsorbed to the protein A layer [see Eq. (4.2.1) in Ref. 1]:

$$
\Delta \Gamma=\Gamma_{m} \Delta h_{l} / h_{m} .
$$

In earlier experiments, a short-term resolution of $\delta \Gamma<1.2 \mathrm{pg} / \mathrm{mm}^{2}$ of the surface mass coverage $\Gamma$ was experimentally demonstrated for $\mathrm{SiO}_{2}$-based sensor chips under stabilized laboratory conditions. ${ }^{1}$ This value corresponds to a resolution of $\delta \mathrm{N}<9.1 \times 10^{-7}$ of the effective refractive index 
Table 1 Stabilization tolerances to reach a resolution $\delta \Gamma$ $<1.2 \mathrm{pg} / \mathrm{mm}^{2}$ for high-sensitivity $\mathrm{TiO}_{2}$ sensor chips based on replicated $\mathrm{PC}$ or $\mathrm{SiO}_{2}$ substrates (without referencing).

\begin{tabular}{lcc}
\hline $\begin{array}{l}\text { Variations } \\
\text { in }\end{array}$ & $\begin{array}{c}\text { PC-based } \\
\text { sensor chips }\end{array}$ & $\begin{array}{c}\mathrm{SiO}_{2} \text {-based } \\
\text { sensor chips }\end{array}$ \\
\hline Temperature & $\delta T<0.015^{\circ} \mathrm{C}$ & $\delta T<0.024^{\circ} \mathrm{C}$ \\
Light wavelength & $\delta \lambda<1.0 \times 10^{-3} \mathrm{~nm}$ & $\delta \lambda<9.5 \times 10^{-4} \mathrm{~nm}$ \\
Angle of incidence & $\delta \theta_{i}<5.2 \times 10^{-5} \mathrm{deg}$ & $\delta \theta_{i}<5.2 \times 10^{-5} \mathrm{deg}$ \\
\hline
\end{tabular}

$N$. Table 1 summarizes the tolerances for stabilization to reach this sensitivity for three distinct external disturbances, namely, for variations of the chip temperature $T$, the light wavelength $\lambda$, and the angle of incidence $\theta_{i}$, if no referencing is used. Calculations have shown that a stabilization of the sensor chip and of all reagents to better than $0.015^{\circ} \mathrm{C}$ for PC-based sensor chips and to better than $0.024{ }^{\circ} \mathrm{C}$ for $\mathrm{SiO}_{2}$-based sensor chips is required for obtaining such high resolution. ${ }^{8}$ The tolerance for the wavelength stabilization was calculated for the same waveguide structures by regarding the materials dispersion. The values for the angle-ofincidence stabilization tolerances result directly from Eq. (1) for $\theta_{i}=0 \mathrm{deg}$.

\section{EXPERIMENTAL RESULTS AND DISCUSSION}

\subsection{SENSING PAD PERFORMANCE}

Figure 5 shows the variations in the sensing pad equivalent thickness for one cycle of an immunoassay, including a regeneration step. The results were obtained for a fused silica-based sensor chip in Figure 5(a) $\left(n_{s}=1.4536\right)$ with a $\mathrm{TiO}_{2}$ waveguiding film $\left(n_{f}=2.3490, h_{f}=159.6 \mathrm{~nm}\right)$ and a grating periodicity $\Lambda=456.0 \mathrm{~nm}$. The same experiments have been performed for a second type of sensor chip based on replicated polycarbonate substrates and coated with a waveguiding $\mathrm{TiO}_{2}$ film using the same lowtemperature deposition process ${ }^{14}$ [Figure 5(b)]. The sensor chip data were $n_{s}=1.571$ for the refractive index of the PC substrate, and $n_{f}=2.3462, h_{f}$ $=137.0 \mathrm{~nm}$ for the refractive index and the thickness of the $\mathrm{TiO}_{2}$ waveguiding film, respectively. The grating periodicity was $\Lambda=451.2 \mathrm{~nm}$. The grating depth of both chips was $h_{g} \approx 5 \mathrm{~nm}$, as measured by atomic force microscopy.

At the beginning of the assay, buffer solution was pumped through the cell at a flow rate of $1 \mathrm{ml} / \mathrm{min}$. In the following step, the sample solution (analyte) containing $10^{-8} \mathrm{Mr}$ r-IgG was supplied to the sensor for about $27 \mathrm{~min}$, resulting in an equivalent adsorbate layer increase of 5470 and $6850 \mathrm{pm}$ for the $\mathrm{SiO}_{2}{ }^{-}$and PC-based sensor chips, respectively. Using an exponential fitting curve, according to the Langmuir theory, an extrapolated saturation value
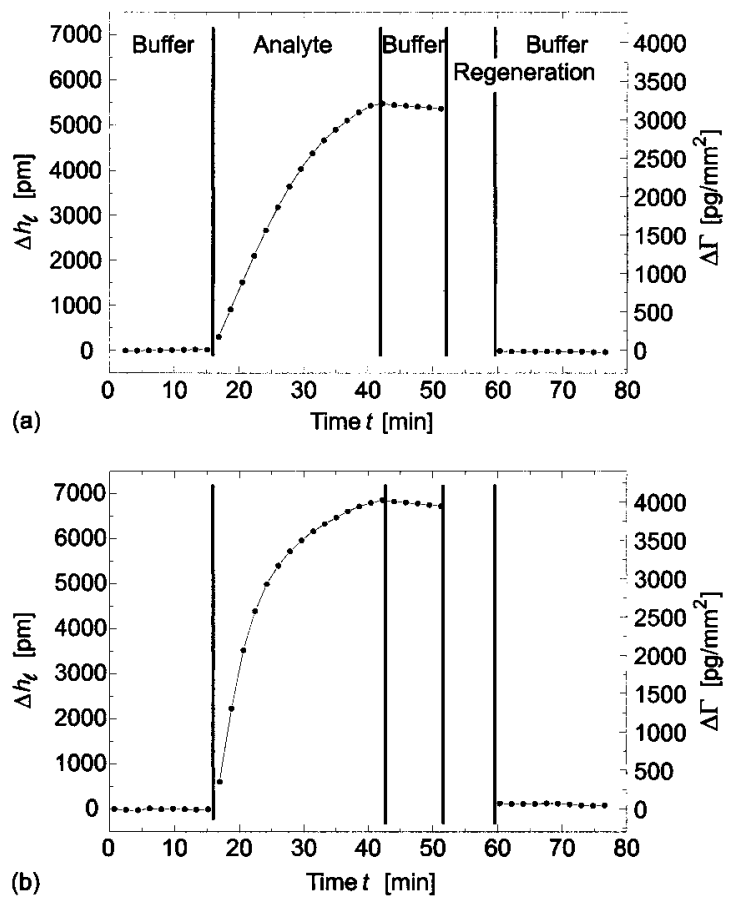

Fig. 5 Sensing layer thickness variations of an immunoassay with a regeneration step. Results obtained for a fused silica- (a) and polycarbonate- (b) based sensor chip. Measurements performed at $\lambda=785.1 \mathrm{~nm}, \mathrm{TM}_{0}$ mode.

of about 6500 and $7000 \mathrm{pm}$ for the two sensor types was calculated, which corresponded to more than three quarters of a monolayer of adsorbed $r-\operatorname{IgG}{ }^{1}$ Generally, this saturation value for the first assay cycle of a sensor chip varied between about 5000 and $7000 \mathrm{pm}$ ( 0.6 to 0.8 of a saturated monolayer) for different sensor chips, independent of the substrate material. The reasons for these variations are being investigated by atomic force microscopy measurements of the sensing layer. After buffer solution was supplied for about $8 \mathrm{~min}$, the regeneration solution (glycine at $\mathrm{pH} 3.4$ ) was supplied to the sensor for about $7 \mathrm{~min}$ (all at a flow rate of $1 \mathrm{ml}$ / min). During this period, no transducer response was shown because it is mainly caused by a change in the bulk refractive index due to the supply of the regeneration solution; therefore the direction of the very fast signal change is only dependent on slight variations of the composition of the solution.

As Figure 5 clearly demonstrates, both sensor chip types exhibited essentially the same behavior and performance. Some differences exist in the temperature dependence, which was 1.6 times larger for the PC-based sensor chips than for the $\mathrm{SiO}_{2}$-based sensor chips, ${ }^{8}$ and in the slightly smaller resolution of the plastic chips due to a $15 \%$ lower sensitivity $\partial \mathrm{N} / \partial h_{l} \cdot{ }^{12}$

In Figure 5(b), the baseline was slightly higher at the end of the assay than at the beginning. Without referencing, it is difficult to find out whether this decrease is an effect that originates from changed 

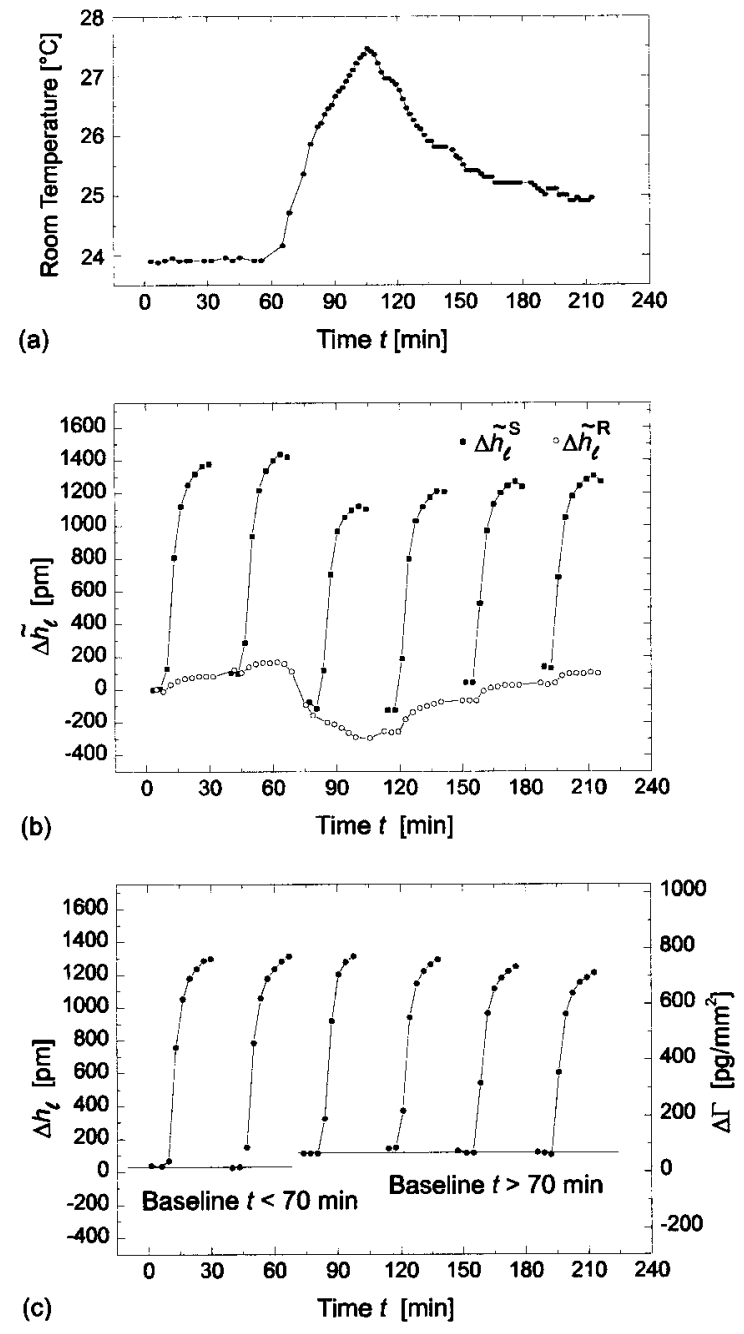

Fig. 6 (a) Variation of the room temperature and resulting (apparent) sensing and reference layer thickness variations for a replicated polycarbonate sensor chip during six consecutive cycles of an immunoassay with a regeneration step. (b) Apparent thickness variations $\Delta \tilde{h}_{l}^{S}(\mathbf{\square})$ and $\Delta \tilde{h}_{l}^{R}(O)$ of the sensing and reference pad, respectively. (c) Calculated equivalent thickness variation $\Delta h_{\text {l }}$ $=\Delta \tilde{h}_{l}^{S}-\Delta \tilde{h}_{l}^{R}$.

sensing layer properties or from external disturbances. Therefore the quantity determined in a nonreferenced assay is not the real one, but an apparent thickness $\tilde{h}_{l}$ of the sensing layer. This is particularly relevant when the experiments are performed under conditions that are not well controlled (e.g., varying room temperature, light wavelength, or angle of incidence).

\subsection{REFERENCING FOR THE TEMPERATURE EFFECT}

The effect of temperature variations on the performance of IO sensor chips has been investigated. Figure 6 shows the results when the room temperature was varied during the experiment as given in Figure 6(a). Furthermore, the temperature of the re- agents was slightly higher than the room temperature at the beginning of the measurement.

Figure 6(b) shows the apparent thickness variations $\Delta \tilde{h}_{l}^{S}$ and $\Delta \tilde{h}_{l}^{R}$ of the sensing and the reference pads, respectively, for six consecutive cycles of an immunoassay. For $t=0$, the apparent thickness variations $\Delta \tilde{h}_{l}^{S}$ and $\Delta \tilde{h}_{l}^{R}$ were set to zero. The results were obtained for another replicated PC sensor chip. The sensor chip data were $n_{f}=2.3492, h_{f}$ $=150.2 \mathrm{~nm}$ for the refractive index and the thickness of the $\mathrm{TiO}_{2}$ waveguiding film, respectively. The grating periodicity was $\Lambda=451.1 \mathrm{~nm}$. The sample solution supply, containing again $10^{-8} \mathrm{M}$ $\mathrm{r}-\mathrm{IgG}$, resulted in an equivalent adsorbate layer increase of about $1400 \mathrm{pm}$ for the first $20 \mathrm{~min}$ while an equivalent change of less than 100 pm was observed on the reference pad. The adsorbate layer increases of the sensing pad were smaller than those shown in Figure 5 because the assay cycles were not the first cycles performed with this sensor chip (see also Sec. 4.5).

The nonconstant room temperature influenced the temperature of the sensor chip and the reagents, which led to an apparent thickness variation of the baselines of the sensing and the reference pad [Figure $6(\mathrm{~b})]$. The response of the reference pad was used to compensate for the disturbance by the temperature effect. Figure 6(c) shows the corrected equivalent thickness variation $\Delta h_{l}=\Delta \tilde{h}_{l}^{S}-\Delta \tilde{h}_{l}^{R}$ of the sensing layer. As can be clearly seen, the baseline is now very stable during the first two assay cycles and during the last four cycles.

In analogy to the term "common mode rejection ratio" (CMRR) often used to describe the performance of electronic operational amplifiers, a "baseline ripple rejection ratio"

$$
\mathrm{BRRR}=\Delta N_{n} / \Delta N_{r}
$$

is an adequate quantity to characterize the performance of IO sensor chips with on-chip referencing. ${ }^{19}$ Here, $\Delta N_{n}$ and $\Delta N_{r}$ denote the baseline variation (ripple) of the effective refractive in$\operatorname{dex} N$ due to temperature effects for nonreferenced and referenced operations, respectively. Using this definition, the results obtained in Figure 6 can be summarized by stating that a $B R R R \approx 10$ was achieved. For the same type of sensor chips, but without any additional sensing layer, a $B R R R \approx 30$ was reported earlier in Ref. 8 . The smaller value for the BRRR achieved in this experiment is probably caused by the asymmetry between the sensing and the reference pad. As an example, temperature variations will induce changes in the sensing and reference layer refractive index and in its thickness, an effect that is not exactly the same for the sensing and reference pad in its present form. As can be seen in Figure 6(c), the sensing layer showed an equivalent thickness increase of about 100 pm during the second assay. The reason for this increase is 

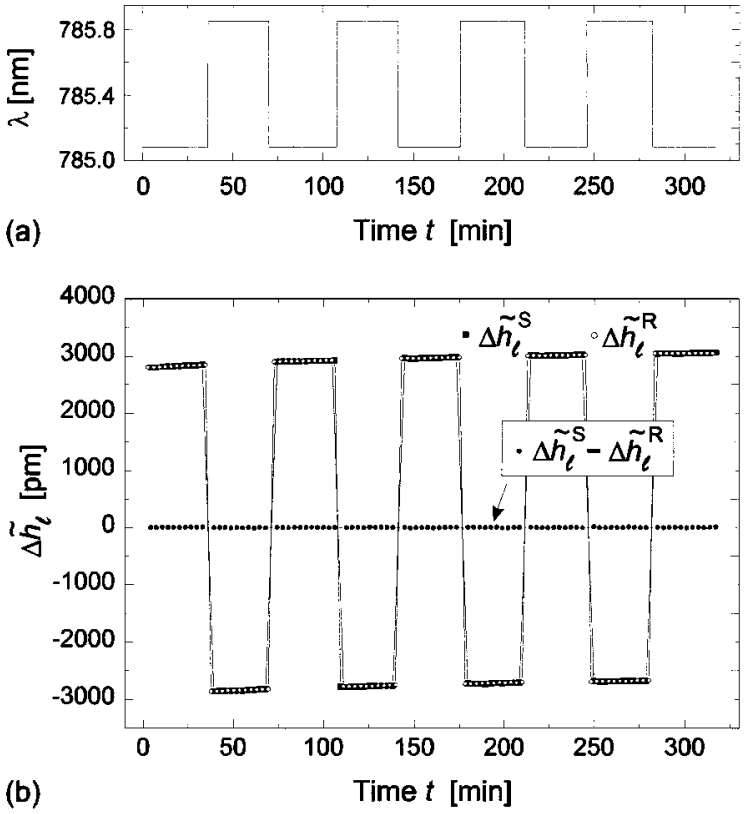

Fig. 7 (a) Wavelength variation of the laser diode. (b) Resulting apparent thickness variations $\Delta \tilde{h}_{l}^{S}(\mathbf{\square})$ and $\Delta \tilde{h}_{l}^{R}(O)$ of the sensing and reference pad, respectively. (0) Calculated equivalent thickness variation $\Delta \tilde{h}_{l}^{S}-\Delta \tilde{h}_{l}^{R}$ for a replicated polycarbonate sensor chip during a buffer sequence.

not known. However, this demonstrates that onchip referencing provides not only the possibility to compensate for the temperature effect but also allows specific sensing layer properties to be investigated in detail.

As mentioned in Sec. 3, a stabilization of the sensor chip and of all reagents to better than $0.015^{\circ} \mathrm{C}$ for PC-based sensor chips and to better than $0.024^{\circ} \mathrm{C}$ for $\mathrm{SiO}_{2}$-based sensor chips is required to obtain a resolution of $\delta \Gamma=1.2 \mathrm{pg} / \mathrm{mm}^{2}$. The experimental results reported here demonstrate that the approach of placing reference pads close to sensing pads leads to a markedly relaxed need for temperature stabilization of about $0.13^{\circ} \mathrm{C}$ for PC-based sensor chips and $0.21^{\circ} \mathrm{C}$ for $\mathrm{SiO}_{2}$-based sensor chips because only the temperature gradient between the sensing and the reference pad is important, and not the absolute temperature.

\subsection{REFERENCING FOR THE WAVELENGTH EFFECT}

Another external effect that affects the performance of IO sensor chips is the disturbance by an unstable detection wavelength $\lambda$. Since the effective index $N$ directly depends on the wavelength, any variations $\Delta \lambda$ of the wavelength induce variations $\Delta N$ of the effective index.

In Figure 7, the transducer response is presented for periodic wavelength variations between $\lambda$ $=785.9$ and $\lambda=785.1 \mathrm{~nm}$ [Figure $7(\mathrm{a})$ ] during a buffer sequence. The wavelength changes were induced by switching the case temperature of the la- ser diode between 25.0 and $27.5^{\circ} \mathrm{C}$, forcing a mode hop of the laser to the next longitudinal mode. Both apparent thickness variations $\Delta \tilde{h}_{l}^{S}(\boldsymbol{\square})$ of the sensing pad and $\Delta \tilde{h}_{l}^{R}(\bigcirc)$ of the reference pad were arbitrarily set to $2800 \mathrm{pm}$ for $t=0$. The calculated equivalent thickness variation $\Delta \tilde{h}_{l}^{S}-\Delta \tilde{h}_{l}^{R}(\bullet)$ reveals a remarkable BRRR of about 350 !

Since the laser diode exhibits a wavelength variation of about $0.06 \mathrm{~nm} /{ }^{\circ} \mathrm{C}$ in a mode hop-free region (which typically corresponds to about a 2 to $4^{\circ} \mathrm{C}$ temperature variation for the type of lasers used in the experiments), on-chip referencing leads to markedly relaxed tolerances for the laser temperature stabilization or even eliminates the need for any stabilization.

In the following paragraphs, experiments are described in which these large disturbances were applied to the sensor while performing a real assay cycle. The immunoassay cycle presented in Figure 8(b) was the first cycle of a whole series performed with the same sensor chip used for the experiments reported in Sec. 4.2.

Figure 8(b) shows the resulting apparent thickness variations $\Delta \tilde{h}_{l}^{S}$ and $\Delta \tilde{h}_{l}^{R}$ of the sensing and the reference pads, respectively. The nonconstant wavelength led to variations of the effective refractive index $N$ of the sensor chip and hence to an apparent thickness variation of the baselines of the sensing and the reference pad [Figure 8(b)]. Figure 8(c) shows the corrected equivalent thickness variation $\Delta h_{l}=\Delta \tilde{h}_{l}^{S}-\Delta \tilde{h}_{l}^{R}$ of the sensing layer. The effect of a not perfectly flat baseline before the analyte supply and of the baseline drop after the regeneration step is discussed in Sec. 4.5. As Figure 8(c) clearly demonstrates, a smooth transducer response curve is obtained in a real experiment even under extremely adverse conditions.

\subsection{REFERENCING FOR THE ANGLE-OF- INCIDENCE EFFECT}

Another external disturbance is the effect of an unstable autocollimation angle of the sensor chip due to instabilities of the mechanical setup. This instability leads to angle-of-incidence variations. Normally, this effect is corrected by averaging the angles of incidence for waveguide modes propagating in opposite directions in the waveguide. Hence, for obtaining a corrected value for the angle of incidence $\theta_{i}$, two angular scans with opposite signs have to be performed. In order to follow fast signal changes, or to improve the quasi-simultaneous readout at the two pads, performance of only one scan is highly desired.

In investigating the influence of variations of the autocollimation angle, results are reported for experiments in which only a single scan was performed to obtain the angle of incidence. In Figure 9, the transducer response is presented for autocollimation angle variations during a buffer sequence. 

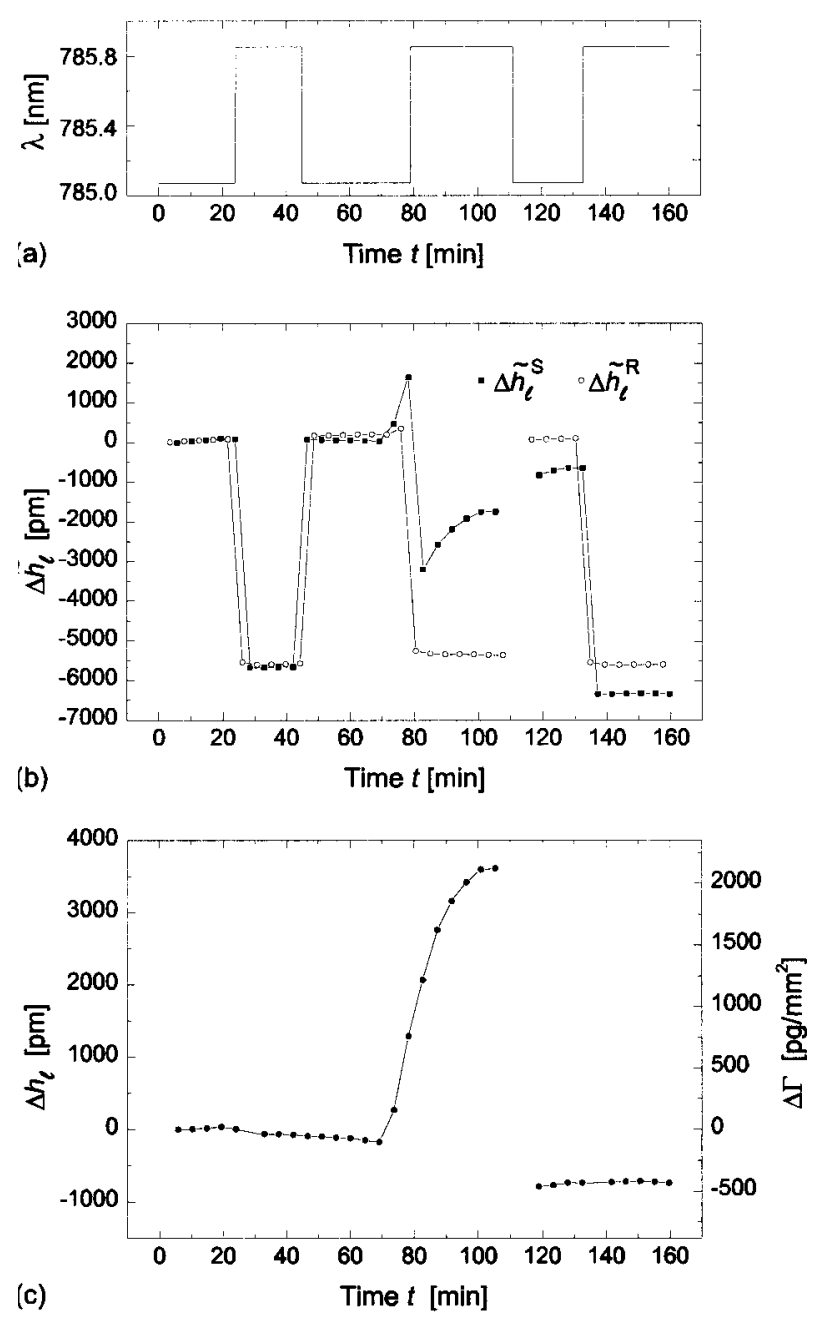

Fig. 8 (a) Wavelength variation of the laser diode and resulting (apparent) sensing and reference layer thickness variations for a replicated polycarbonate sensor chip during one cycle of the immunoassay with a regeneration step. (b) Apparent thickness variations $\Delta \tilde{h}_{l}^{S}(\mathbf{\square})$ and $\Delta \tilde{h}_{l}^{R}(O)$ of the sensing and reference pad, respectively. (c) Calculated equivalent thickness variation $\Delta h_{l}=$ $\Delta \tilde{h}_{l}^{S}-\Delta \tilde{h}_{l}^{R}$.

Both apparent thickness variations $\Delta \tilde{h}_{l}^{S}(\mathbf{\square})$ of the sensing pad and $\Delta \tilde{h}_{l}^{R}(\bigcirc)$ of the reference pad were arbitrarily set to $500 \mathrm{pm}$ for $t=0$.

In this experiment, the calculated equivalent thickness variation $\Delta \tilde{h}_{l}^{S}-\Delta \tilde{h}_{l}^{R}(\bullet)$ corresponds to a BRRR of about 100. Hence, the immunity against mechanical disturbances is significantly enhanced to a tolerance of $\delta \theta_{i}<5.2 \times 10^{-3} \mathrm{deg}(=18.7$ arc seconds).

While performing an assay cycle with the same chip, the autocollimation angle of the experimental setup was deliberately disadjusted as plotted in Figure 10(a). Figure 10(b) shows the resulting apparent thickness variations $\Delta \tilde{h}_{l}^{S}$ and $\Delta \tilde{h}_{l}^{R}$ of the sensing and the reference pads, respectively. The nonconstant autocollimation angle led to incorrect values for the angle of incidence $\theta_{i}$, yielding appar-
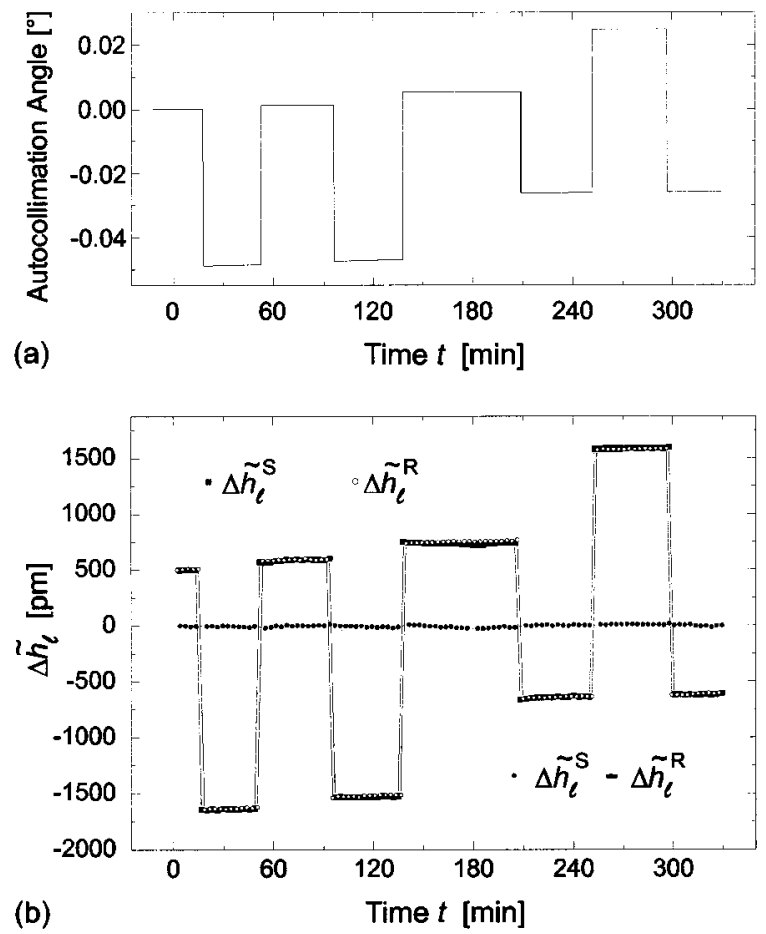

Fig. 9 (a) Autocollimation angle variation. (b) Resulting apparent thickness variations $\Delta \tilde{h}_{l}^{S}(\mathbf{\square})$ and $\Delta \tilde{h}_{l}^{R}(O)$ of the sensing and reference pad, respectively. Calculated equivalent thickness variation $\Delta \tilde{h}_{l}^{S}-\Delta \tilde{h}_{l}^{R}(\bullet)$ for a replicated polycarbonate sensor chip during a buffer sequence.

ent variations of the sensing layer thickness [according to Eq. (1)]. Figure 10(c) shows the corrected equivalent thickness variation $\Delta h_{l}=\Delta \tilde{h}_{l}^{S}-\Delta \tilde{h}_{l}^{R}$ of the sensing layer, clearly demonstrating that a smooth transducer response curve is obtained even for a very unstable angle of incidence.

\subsection{CONSECUTIVE ASSAY CYCLES}

The influence of consecutive assay cycles on the two pads has been investigated in a series of repetitive experiments. Figure 11(a) shows the apparent thickness variations $\Delta \tilde{h}_{l}^{S}$ and $\Delta \tilde{h}_{l}^{R}$ of the sensing and the reference pads, respectively, for another replicated PC sensor chip but with the same chip data as given in Sec. 4.2. In Figure 11(b), the corrected equivalent thickness variations $\Delta h_{l}$ are presented. During the first two cycles, $10^{-8} M r$-IgG was supplied to the sensor; then the analyte concentration was alternated between $10^{-8}$ and $10^{-9} \mathrm{M}$. In each cycle, the analyte was supplied for $20 \mathrm{~min}$, and the duration of a full cycle, including regeneration, was about $33 \mathrm{~min}$. The equivalent increase of the surface coverage by bound r-IgG after the association step dropped to about one fifth after 31 cycles over a measuring period of $17 \mathrm{hr}$ and a total amount of about $1200 \mathrm{ml}$ reagent solution supplied to the sensor. Supplying less aggressive re- 

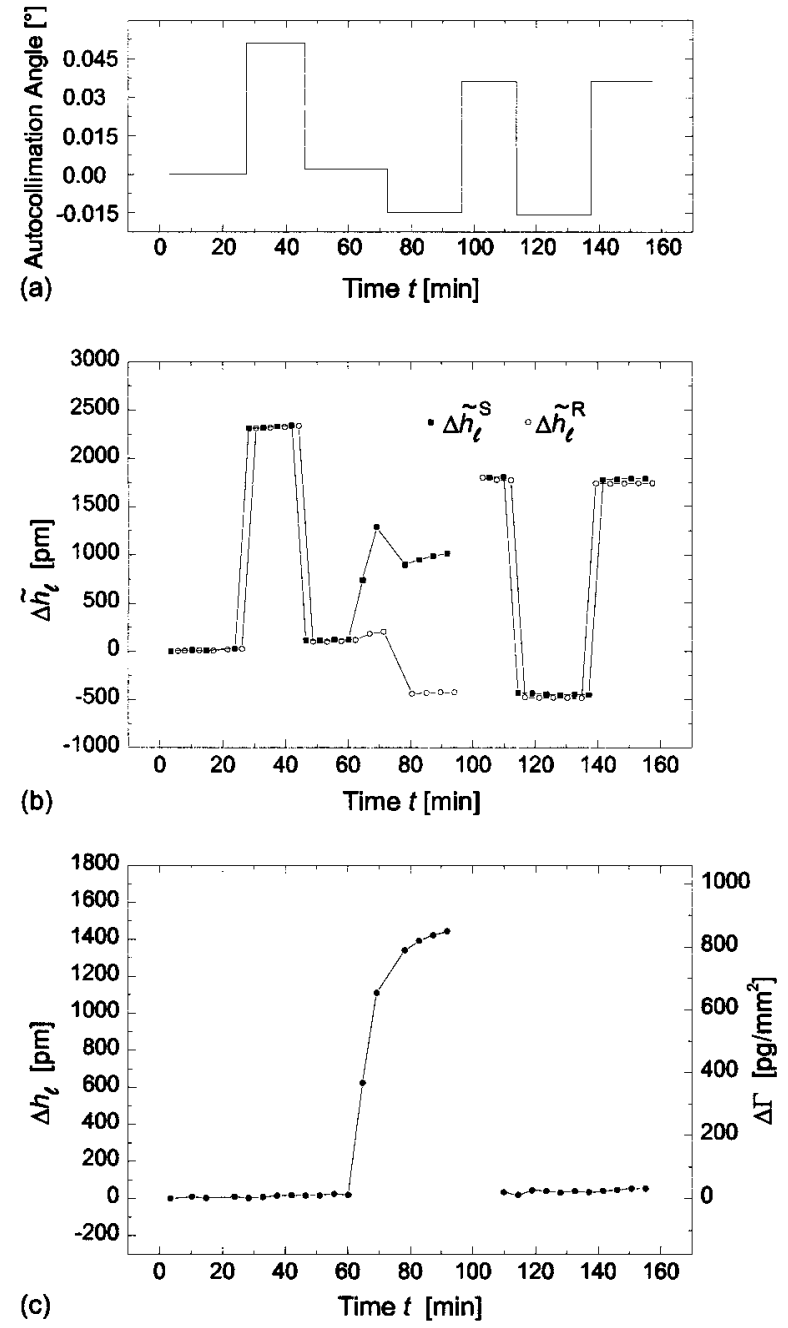

Fig. 10 (a) Autocollimation angle variation and resulting (apparent) sensing and reference layer thickness variations for a replicated polycarbonate sensor chip during one cycle of the immunoassay with a regeneration step. (b) Apparent thickness variations $\Delta \tilde{h}_{l}^{S}(\mathbf{\square})$ and $\Delta \tilde{h}_{l}^{R}(O)$ of the sensing and reference pad, respectively. (c) Calculated equivalent thickness variation $\Delta h_{l}=$ $\Delta \tilde{h}_{l}^{S}-\Delta \tilde{h}_{l}^{R}$.

generation solution at higher $\mathrm{pH}$ may improve this signal reduction. The marked drop of the baseline during the first four consecutive assay cycles is probably due to removed protein $A$ [see Figure 11(b) and Figure 8(c)]. It was also observed [see Figure $8(\mathrm{c})$ ] that the baseline slightly decreased before the sensor was regenerated for the first time. The reason for this effect is probably some protein $A$ that was not properly immobilized and therefore slowly washed away when buffer was supplied for the first time.

As can be seen in Figure 11(a), the functionality of the sensing and the reference pads is still excellent after this long measuring period: even the $10^{-9} \mathrm{M}$ r-IgG analyte supply yields a transducer response that is more than two orders of magnitude larger than the resolution of the experimental setup [see inset in Figure 11(b)].
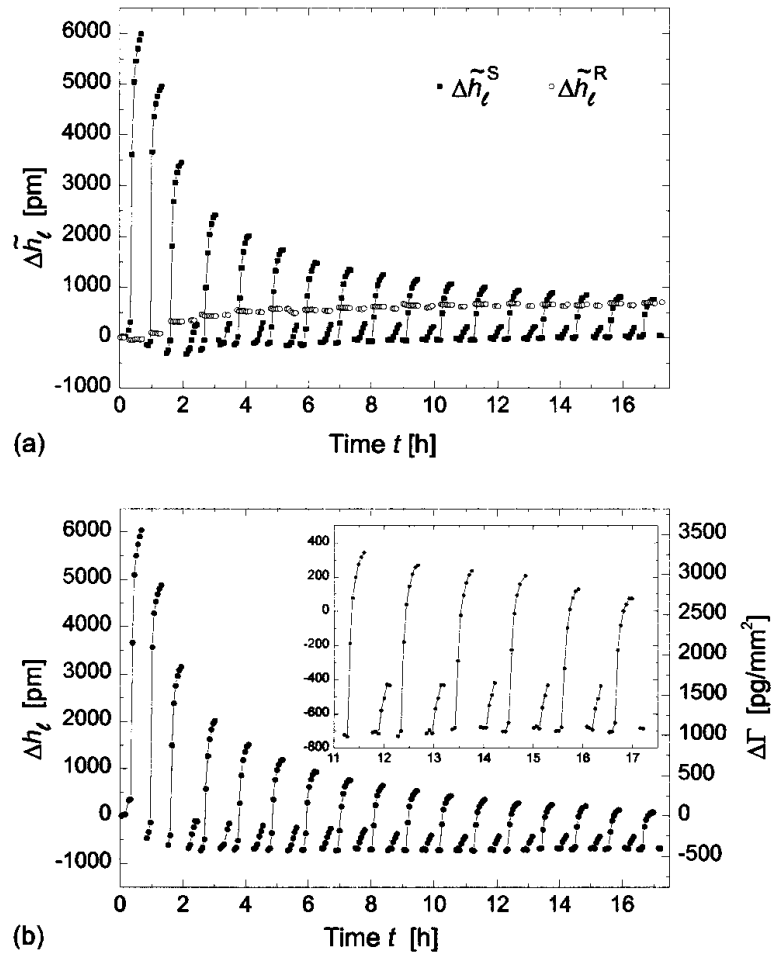

Fig. 11 Sensing and reference layer thickness variations for a replicated polycarbonate sensor chip during 31 cycles of an immunoassay with a regeneration step. (a) Apparent thickness variations $\Delta \tilde{h}_{l}^{S}(\mathbf{\square})$ and $\Delta \tilde{h}_{l}^{R}(O)$ of the sensing $S$ and reference pad $R$, respectively. (b) Calculated equivalent thickness variation $\Delta h_{l}=$ $\Delta \tilde{h}_{l}^{S}-\Delta \tilde{h}_{l}^{R}(\mathbf{O})$. The inset in (b) shows a detailed view for $t=11$ to $17 \mathrm{hr}$.

\subsection{ADVANTAGES OF ON-CHIP REFERENCING}

The experimental results reported in this section demonstrate that placing reference pads close to sensing pads leads to markedly relaxed tolerances for stabilization, and allows the study of specific sensing layer properties in more detail. The relaxed stabilization requirements are important for the development of sensor systems for practical applications. Furthermore, the high resolution can be maintained over a prolonged duration, yielding an improved detection limit.

Table 2 summarizes the stabilization tolerances for the experiments and sensor chips presented in this paper. Although the reference pad was not optimized for this type of experiment, a marked improvement was clearly achieved by on-chip referencing. Even more relaxed tolerances can be expected when more effort is taken to develop reference pads that minimize the asymmetry to the sensing pad with respect to the dominant disturbance. 
Table 2 Comparison of stabilization tolerances to reach a resolution $\delta \Gamma<1.2 \mathrm{pg} / \mathrm{mm}^{2}$ for $\mathrm{TiO}_{2}$ sensor chips based on replicated PC or $\mathrm{SiO}_{2}$ substrates, without and with referencing.

\begin{tabular}{|c|c|c|c|c|}
\hline \multirow[b]{2}{*}{ Variations in } & \multicolumn{2}{|c|}{ Without referencing } & \multicolumn{2}{|c|}{ With on-chip referencing } \\
\hline & PC chips & $\mathrm{SiO}_{2}$ chips & PC chips & $\mathrm{SiO}_{2}$ chips \\
\hline Temperature & $\delta T<0.015^{\circ} \mathrm{C}$ & $\delta T<0.024^{\circ} \mathrm{C}$ & $\delta T<0.13^{\circ} \mathrm{C}$ & $\delta T<0.21^{\circ} \mathrm{C}$ \\
\hline Light wavelength & $\delta \lambda<1.0 \times 10^{-3} \mathrm{~nm}$ & $\delta \lambda<9.5 \times 10^{-4} \mathrm{~nm}$ & $\delta \lambda<0.35 \mathrm{~nm}$ & $\delta \lambda<0.33 \mathrm{~nm}$ \\
\hline Angle of incidence & $\delta \theta_{i}<5.2 \times 10^{-5} \mathrm{deg}$ & $\delta \theta_{i}<5.2 \times 10^{-5} \mathrm{deg}$ & $\delta \theta_{i}<5.2 \times 10^{-3} \mathrm{deg}$ & $\delta \theta_{i}<5.2 \times 10^{-3} \mathrm{deg}$ \\
\hline
\end{tabular}

\section{CONCLUSIONS}

An experimental setup and a procedure for performing on-chip referencing experiments has been presented. The usefulness of placing reference pads close to sensing pads was rendered obvious for compensating for variations of the temperature, the wavelength, and the angle of incidence of a simple immunoassay model system.

For the temperature effect, the improvement in baseline stability relaxes the tolerances for temperature stabilization of the sensor and all the liquids supplied to the test cell by a factor of about 10 because only the temperature gradient between the sensing and reference pad is important and no longer the absolute temperature. The reason this factor is not larger lies probably in the asymmetry of the thermal properties of the actual sensing and reference pads. For the wavelength and angle-ofincidence effect, large baseline ripple rejection ratios of about 350 and 100 were achieved, respectively. The experiments have impressively shown that if on-chip referencing is used, clean and smooth shapes of the assay curves can be obtained even for assay cycles performed under adverse conditions.

Experiments with consecutive assay cycles showed that the functionality of the sensing and the reference pads remains excellent for more than 30 cycles and a measuring period of 1 day. Even after this time, the supply of $10^{-9} \mathrm{Mr}$ r-IgG analyte yields a transducer response that is well defined and far above the detection limit.

In all experiments, no significant differences for polycarbonate-based sensor chips compared to fused silica sensor chips were found. Replicated polycarbonate sensor chips, which can be mass produced at low cost, are thus suitable for creating miniature sensor modules for applications requiring high-performance disposable chips.

The experimental results clearly demonstrate that on-chip referencing is essential for practical sensors with high sensitivity for compensating or correcting for external disturbances and nonspecific effects. On-chip referencing also allows specific sensing layer properties to be investigated, for example, instabilities of (bio-)chemical layers.

\section{Acknowledgments}

We gratefully thank M. T. Gale, R. Stutz, and S. Westenhöfer for their support in chip fabrication; M. Kuhn and M. Wiki for help with the experimental setup; and J. Edlinger of Balzers, Liechtenstein, for depositing the waveguiding films. This work was partially supported by the Schweizerische Stiftung für mikrotechnische Forschung (FSRM, No. 92/07) and the Kommission für Technologie und Innovation (KTI, No. 2844.2).

\section{REFERENCES}

1. R. E. Kunz, G. Duveneck, and M. Ehrat, "Sensing pads for hybrid and monolithic integrated optical immunosensors," Proc. SPIE 2331, 2-17 (1994).

2. R. G. Heideman, R. P. H. Kooyman, and J. Greve, "Performance of a highly sensitive optical waveguide MachZehnder interferometer immunosensor," Sensors Actuators B 10, 209-217 (1993).

3. D. Schlatter, R. Barner, Ch. Fattinger, W. Huber, J. Hübscher, J. Hurst, H. Koller, C. Mangold, and F. Müller, "The difference interferometer: application as a direct affinity sensor," Biosensors Bioelectronics 8, 109-116 (1993).

4. Ch. Stamm and W. Lukosz, "Integrated optical difference interferometer as biochemical sensor," Sensors Actuators B 18-19, 183-187 (1994).

5. W. Lukosz, D. Clerc, and Ph.M. Nellen, "Input and output grating couplers as integrated optical biosensors," Sensors Actuators A 25-27, 181-184 (1991).

6. A. Brandenburg, R. Polzius, F. Bier, U. Bilitewski, and E. Wagner, "Direct observation of affinity reactions by reflected-mode operation of integrated optical grating coupler,' Sensors Actuators B 30, 55-59 (1996).

7. D. Freiner, R. E. Kunz, D. Citterio, U. E. Spichiger, and M. T. Gale, "Integrated optical sensors based on refractometry of ion-selective membranes," Sensors Actuators B 29, 277-285 (1995).

8. J. Dübendorfer and R. E. Kunz, "Reference pads for miniature integrated optical sensors," Sensors Actuators B 38, 116121 (1997).

9. J. Dübendorfer, R. E. Kunz, E. Mader, G. L. Duveneck, and M. Ehrat, "Reference and sensing pads for integrated optical immunosensors," Proc. SPIE 2928, 90-97 (1996).

10. J. Ingenhoff, B. Drapp, and G. Gauglitz, "Biosensors using integrated optical devices," Fresenius J. Anal. Chem. 346, 580583 (1993).

11. D. S. Smith, M. H. Al-Hakiem, and H. Landon, "A review of fluoroimmunoassay and immunofluorometric assay," J. Ann. Clin. Biochem. 18, 253-274 (1981).

12. R. E. Kunz, J. Dübendorfer, and R. H. Morf, "Finite grating depth effects for integrated optical sensors with high sensitivity," Biosensors Bioelectronics 11, 653-667 (1996).

13. R. E. Kunz, J. Edlinger, B. J. Curtis, M. T. Gale, L. U. Kempen, H. Rudigier, and H. Schütz, "Grating couplers in 
tapered waveguides for integrated optical sensing," Proc. SPIE 2068, 313-325 (1994).

14. R. E. Kunz, J. Edlinger, P. Sixt, and M. T. Gale, "Replicated chirped waveguide gratings for optical sensing applications," Sensors Actuators A 47, 482-486 (1995).

15. D. W. Hewak and J. W. Y. Lit, "Generalized dispersion properties of a four-layer thin-film waveguide," Appl. Opt. 26/5, 833-841 (1987).

16. K. Tiefenthaler and W. Lukosz, "Sensitivity of grating couplers as integrated-optical chemical sensors," J. Opt. Soc. Am. B 6, 209-220 (1989).
17. E. Anemogiannis, E. N. Glytsis, and T. K. Gaylord, "Optimization of multilayer integrated optics waveguides," J. Lightwave Technol. 12/3, 512-517 (1994).

18. Ph. M. Nellen and W. Lukosz, "Integrated optical input grating couplers as direct affinity sensors," Biosensors Bioelectronics 8, 129-147 (1994).

19. R. E. Kunz, "Miniature integrated optical modules for chemical and biochemical sensing," Sensors Actuators B 38, 13-28 (1997). 